\title{
NON-ABELIAN EXTERIOR PRODUCTS OF GROUPS AND EXACT SEQUENCES IN THE HOMOLOGY OF GROUPS
}

\author{
by G. J. ELLIS $\dagger$
}

(Received 4 December, 1984)

0. Introduction. Various authors have obtained an eight term exact sequence in homology

$$
H_{3}(G) \rightarrow H_{3}(Q) \rightarrow V \rightarrow H_{2}(G) \rightarrow H_{2}(Q) \rightarrow N /[G, N] \rightarrow H_{1}(G) \rightarrow H_{1}(Q) \rightarrow 0
$$

from a short exact sequence of groups

$$
1 \rightarrow N \rightarrow G \stackrel{\Phi}{\rightarrow} Q \rightarrow 1,
$$

the term $V$ varying from author to author (see [7] and [2]; see also [5] for the simpler case where $N$ is central in $G$, and [6] for the case where $N$ is central and $N \subset[G, G])$. The most satisfying version of the sequence is obtained by Brown and Loday [2] (full details of [2] are in [3]) as a corollary to their van Kampen type theorem for squares of spaces: they give the term $V$ as the kernel of a map $G \wedge N \rightarrow N$ from a "non-abelian exterior product" of $G$ and $N$ to the group $N$ (the definition of $G \wedge N$, first published in [2], is recalled below). The two short exact sequences

$$
1 \rightarrow G \rightarrow G \rightarrow 1 \rightarrow 1
$$

and

$$
1 \rightarrow R \rightarrow F \rightarrow G \rightarrow 1
$$

where $F$ is free, together with the fact that $H_{2}(F)=0$ and $H_{3}(F)=0$, imply isomorphisms

$$
\begin{gathered}
H_{2}(G) \cong \operatorname{ker}(G \wedge G \rightarrow G), \\
H_{3}(G) \cong \operatorname{ker}(F \wedge R \rightarrow R) .
\end{gathered}
$$

The isomorphism (2) is essentially the description of $H_{2}(G)$ proved algebraically in [11]. As noted in [2], the isomorphism (3) is the analogue for $H_{3}(G)$ of the Hopf formula for $H_{2}(G)$.

The aim of this paper is to give an algebraic proof of the isomorphism (3), and then to use the isomorphisms (2) and (3) to give a purely algebraic derivation of the (Brown and Loday version of) exact sequence (1). Further, we shall show that (1) is part of a long exact sequence. More precisely, denote by $B(G)_{\#}, B(Q)_{\#}$ the bar resolutions of $G$ and $Q$ over $\mathbf{Z}$. The surjection $\phi: G \rightarrow Q$ gives rise to a short exact sequence of chain complexes

$$
0 \rightarrow \operatorname{ker} \phi_{\#} \rightarrow B(G)_{\#} \otimes_{G} \mathbf{Z} \stackrel{\phi_{*}}{\rightarrow} B(Q)_{\#} \otimes_{Q} \mathbf{Z} \rightarrow 0
$$

† The contents of this paper form part of the author's Ph.D. thesis which was supervised by Prof. R. Brown.

Glasgow Math. J. 29 (1987) 13-19. 
which in turn gives rise to a long exact homology sequence

$$
\rightarrow H_{n}\left(\operatorname{ker} \phi_{\#}\right) \rightarrow H_{n}(G) \rightarrow H_{n}(Q) \rightarrow H_{n-1}\left(\operatorname{ker} \phi_{\sharp}\right) \rightarrow \text {. }
$$

We shall give isomorphisms

$$
\begin{gathered}
H_{1}\left(\operatorname{ker} \phi_{\#}\right) \cong N /[G, N], \\
H_{2}\left(\operatorname{ker} \phi_{\#}\right) \cong \operatorname{ker}(G \wedge N \rightarrow N) .
\end{gathered}
$$

The extension of (1) to a long exact sequence is also clear from the topological approach taken in $[2,3]$ (see Remark 1 below and also [1]).

One purpose of giving an algebraic derivation of (1) is to find similar results in other contexts. This will be done for Lie algebras in a sequel.

1. The "non-abelian" exterior product. Let $M, N$ be normal subgroups of a group $G$. The exterior product of $M$ and $N$ is the group $M \wedge N$ generated by the elements $m \wedge n$ with $(m, n) \in M \times N$, subject to the relations

$$
\begin{gathered}
m m^{\prime} \wedge n={ }^{m}\left(m^{\prime} \wedge n\right)(m \wedge n), \\
m \wedge n n^{\prime}=(m \wedge n)^{n}\left(m \wedge n^{\prime}\right), \\
m \wedge n=1 \text { whenever } m=n .
\end{gathered}
$$

where by definition ${ }^{x}(y \wedge z)=\left({ }^{x} y \wedge{ }^{x} z\right)=\left(x y x^{-1} \wedge x z x^{-1}\right)$, and conjugation ${ }^{x} y=x y x^{-1}$ is taken in the group $G$. (A more general construction $M^{\prime} \wedge N^{\prime}$ is given in [2] for arbitrary crossed $G$-modules $M^{\prime}, N^{\prime}$.)

The exterior product $M \wedge N$ can also be defined by its universal property: given a group $H$ and a function $h: M \times N \rightarrow H$, we say that $h$ is an exterior pairing if for all $m, m^{\prime} \in M, n, n^{\prime} \in N$,

$$
\begin{gathered}
h\left(m m^{\prime}, n\right)=h\left({ }^{m} m^{\prime},{ }^{m} n\right) h(m, n), \\
h\left(m, n n^{\prime}\right)=h(m, n) h\left({ }^{n} m,{ }^{n} n^{\prime}\right), \\
h(m, n)=1 \text { whenever } m=n ;
\end{gathered}
$$

the function $M \times N \rightarrow M \wedge N,(m, n) \mapsto m \wedge n$ is the universal exterior pairing from $M \times N$.

It is perhaps helpful to note that the function $M \times N \rightarrow M \cap N,(m, n) \mapsto[m, n]=$ $m n m^{-1} n^{-1}$ is an example of an exterior pairing.

We shall now give some results on the exterior product.

Suppose that $K$ is a normal subgroup of $G$ which is contained in the intersection $M \cap N$. The exterior pairing $K \times N \rightarrow M \wedge N,(k, n) \mapsto k \wedge n$ induces a homomorphism $\iota_{1}: K \wedge N \rightarrow M \wedge N$, and the exterior pairing $M \times K \rightarrow M \wedge N,(m, k) \mapsto m \wedge k$ induces a homomorphism $\iota_{2}: M \wedge K \rightarrow M \wedge N$. Let $K_{1}=\iota_{1}(K \wedge N)$ and $K_{2}=\iota_{2}(M \wedge K)$. The group product $K_{1} K_{2}$ is clearly a normal subgroup of $M \wedge N$ and we have

Proposition 1. There is a canonical isomorphism $(M / K) \wedge(N / K) \cong M \wedge N / K_{1} K_{2}$. 
Proof. The proof is a routine verification of the universal property of the exterior product which we leave to the reader.

Any group $G$ can be considered as a normal subgroup of itself, and so the exterior product $G \wedge G$ can always be constructed. It is a simple matter to check that $G \wedge G$ is isomorphic to the group $\langle G, G\rangle / B(G)$ introduced by Miller [11]. Theorem 1 of that paper can be restated as

Proposition 2 (Miller). If $F$ is a free group, then the homomorphism $\partial: F \wedge F \rightarrow$ $[F, F], f \wedge f^{\prime} \mapsto\left[f, f^{\prime}\right]$ is an isomorphism.

Proof. Let $\pi_{3}(F \wedge F)$ denote the kernel of the homomorphism $\partial: F \wedge F \rightarrow[F, F]$. The key step in Miller's proof is the fact [11, p. 398] that if $F=A * B$ is a free product then $\pi_{3}(F \wedge F)=\pi_{3}(A \wedge A) \times \pi_{3}(B \wedge B)$. The result then follows easily.

As a consequence of Proposition 2 we have

COROLLARY 3. If $F$ is a free group and $R \stackrel{\iota}{\rightarrow} F$ the inclusion of a normal subgroup $R$, then the canonical homomorphisms $\iota \wedge 1: R \wedge R \rightarrow F \wedge R, \iota \wedge \iota: R \wedge R \rightarrow F \wedge F$ are normal inclusions.

Proof. The commutative diagrams

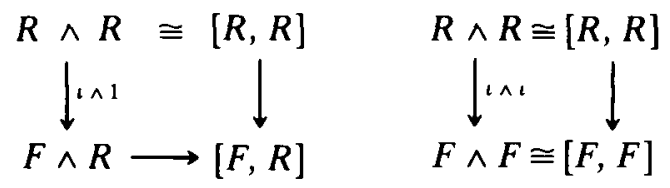

imply the injectivity of the homomorphisms $\iota \wedge 1$ and $\iota \wedge \iota$. For all $f \in F, r, r^{\prime}, r^{\prime \prime} \in R$, the following identity (in both $F \wedge R$ and $F \wedge F$ ) is immediately derivable from proposition 4.1(d) of [2]:

$$
(f \wedge r)\left(r^{\prime} \wedge r^{\prime \prime}\right)(f \wedge r)^{-1}=\left({ }^{[f, r]} r^{\prime} \wedge{ }^{[f, r]} r^{\prime \prime}\right) .
$$

It follows from this identity that the inclusions $\iota \wedge 1, \iota \wedge \iota$ are normal.

We shall use the inclusions of Corollary 3 to regard $R \wedge R$ as a subgroup of $F \wedge R$ and of $F \wedge F$.

Proposition 4. If $R \rightarrow F \stackrel{\rho}{\rightarrow} G$ is a free presentation of the group $G$, then there is a short exact sequence of groups

$$
1 \rightarrow[R, R] \rightarrow F \wedge R \stackrel{\psi}{\rightarrow} I G \otimes_{G} R^{\mathrm{ab}} \rightarrow 0,
$$

where $I G$ is the augmentation ideal of $G$ and $\otimes_{G}$ denotes the usual tensor product of $G$-modules. 
Proof. The map $\psi$ is given on generators by $\psi(f \wedge r)=(\rho f-1) \otimes \bar{r}$ where $\bar{r}$ is the element of $R^{\mathrm{ab}}$ represented by $r$; it is readily checked that $\psi$ is well defined. The group $I G \otimes_{G} R^{\text {ab }}$ is generated by the elements $(g-1) \otimes \bar{r}$ with $g \in G, \bar{r} \in R^{\text {ab }}$. Plainly $\psi$ is surjective. The composite map $[R, R] \stackrel{\cong}{\longrightarrow} R \wedge R \stackrel{\llcorner\wedge 1}{\longrightarrow} F \wedge R$ is injective by Corollary 3 . To complete the proof we need to show that the homomorphism $\psi^{\prime}: F \wedge R / R \wedge R \rightarrow$ $I G \otimes_{G} R^{\mathrm{ab}}$ induced by $\psi$ is an isomorphism.

For all $f, f^{\prime} \in F, r, r^{\prime} \in R$, the following two identities in $F \wedge R$ are immediately derivable from Proposition 4.1 of [2]:

$$
\begin{gathered}
r^{\prime}(f \wedge r)^{-1}(f \wedge r)^{-1}=r^{\prime} \wedge[f, r], \\
{\left[(f \wedge r),\left(f^{\prime} \wedge r^{\prime}\right)\right]=[f, r] \wedge\left[f^{\prime}, r^{\prime}\right] .}
\end{gathered}
$$

The identity (11) implies that the group $T=F \wedge R / R \wedge R$ is abelian. Suppose that $(x, \bar{r})$ is an arbitrary element of the cartesian product $I G \times R^{\mathrm{ab}}$. Thus $x$ can be written as a sum $x=\sum \varepsilon_{i}\left(g_{i}-1\right)$ with $g_{i} \in G, \varepsilon_{i}= \pm 1$. Let $\theta: I G \times R^{a b} \rightarrow T$ be the function which sends $(x, \bar{r})$ to the coset of $\Pi\left(\tilde{g}_{i} \wedge r\right)^{\varepsilon_{i}}$ in $T$, where $\rho \tilde{g}_{i}=g_{i}$. The function $\theta$ is well defined since $T$ is abelian and since, for all $g \in G, r, r^{\prime}, r^{\prime \prime} \in R$, it can be shown that

and, by (10)

$$
\tilde{g} r \wedge r^{\prime} \equiv\left(\tilde{g} \wedge r^{\prime}\right) \quad \text { modulo } R \wedge R \text {, }
$$

$$
\tilde{g} \wedge r\left[r^{\prime}, r^{\prime \prime}\right] \equiv(\tilde{g} \wedge r) \quad \text { modulo } R \wedge R \text {. }
$$

Using (10) again one sees that $\theta$ is a $G$-bilinear map. Hence it induces a homomorphism $\theta^{\prime}: I G \otimes_{G} R^{\mathrm{ab}} \rightarrow T$. It is readily checked that $\theta^{\prime}$ is an inverse to the homomorphism $\psi^{\prime}$ and the proof is complete.

2. The second and third homology of a group. Throughout this section let us suppose given a commutative diagram of groups in which the rows and columns are exact and $F$ is free; such a diagram can be constructed for any surjective homomorphism $\phi$.

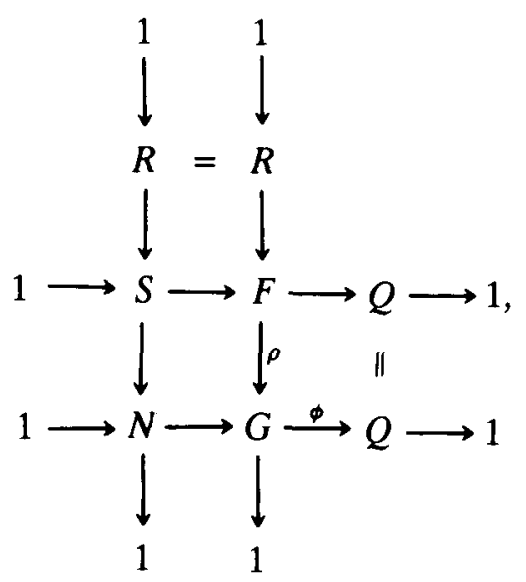

Let us define the group $\pi_{3}(M \wedge N)$ to be the kernel of the homomorphism $\partial: M \wedge N \rightarrow M \cap N, m \wedge n \mapsto[m, n]$. This notation is in keeping with the topological significance of the exterior product (see remark 1 below and $[2,3,4,9]$ ). 
THEOREM 5. There is an isomorphism $H_{2}(G) \cong \pi_{3}(G \wedge G)$.

Proof. It follows from Propositions 1 and 2 that $G \wedge G$ is isomorphic to $[F, F] /[F, R]$. The theorem now follows from the Hopf formula

$$
H_{2}(G) \cong R \cap[F, F] /[F, R] .
$$

Theorem 5 is given in [2] and, modulo a few formal differences, in [11]. Our proof is a slight modification of the one given in [11]. The following result is obtained in [2] by topological methods.

THEOREM 6. There is an isomorphism $H_{3}(G) \cong \pi_{3}(F \wedge R)$.

Proof. Let $\delta: I G \otimes_{G} R^{\mathrm{ab}} \rightarrow R^{\mathrm{ab}}$ be the homomorphism $(g-1) \otimes \bar{r} \mapsto \overline{\left(\tilde{g} r \tilde{g}^{-1} r^{-1}\right)^{-}}$. Then $H_{3}(G) \cong H_{1}\left(G, R^{\text {ab }}\right) \cong \operatorname{ker} \delta$ (see [8, Chapter VI, sections 4 and 12]). We thus have a commutative diagram with exact rows and columns

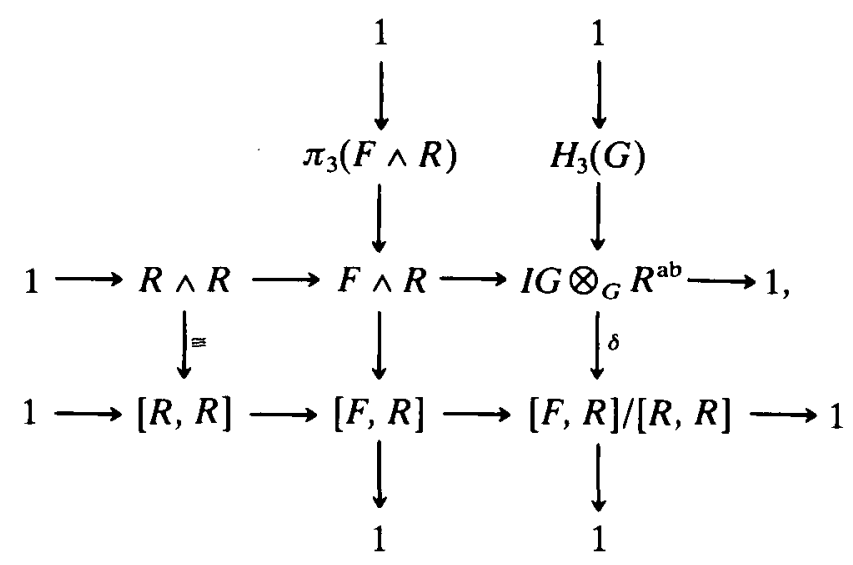

from which the theorem follows.

Recall from the Introduction that ker $\phi_{\#}$ is defined to be the kernel of a chain map induced by the homomorphism $\phi$.

THEOREM 7. There is a commutative diagram

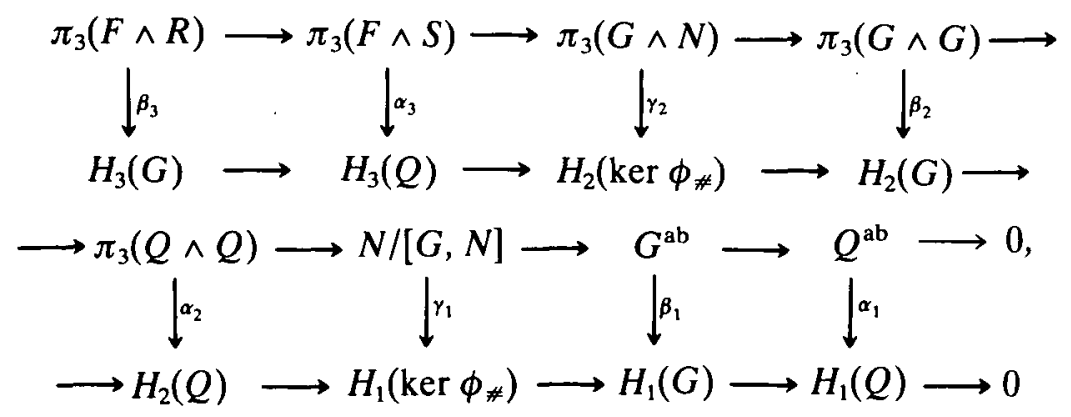

in which the rows are exact and the vertical maps are isomorphisms. 
Proof. The maps in the top row are canonical. Proposition 1 implies isomorphisms $Q \wedge Q \cong G \wedge G / \iota_{2}(G \wedge N), \quad G \wedge G \cong F \wedge F / \iota_{2}(F \wedge R)$ and $G \wedge N \cong F \wedge S / \iota_{2}(F \wedge R)$. Armed with these isomorphisms, it is routine to check the exactness of the top row. The maps $\alpha_{1}, \beta_{1}$ are the standard isomorphisms; the maps $\alpha_{2}, \beta_{2}$ are the isomorphisms of Theorem 5, and the maps $\alpha_{3}, \beta_{3}$ are the isomorphisms of Theorem 6. (Note that at this point we have proved the existence of the exact sequence (1).) The bottom row is obtained as in the Introduction.

Recall that if we take $F$ to be the free group on the set $G \backslash 1$, then the bar resolution $B(G)_{\#}$ has a description in which $B(G)_{2 n}=\mathbf{Z} G \otimes_{\mathbf{Z}}\left(R^{\text {ab }}\right)^{n}, \quad B(G)_{2 n+1}=$ $\left(\mathbf{Z} G \otimes_{F} I F\right) \otimes_{\mathbf{Z}}\left(R^{\mathrm{ab}}\right)^{n}$ where $\mathbf{Z} G$ is the group ring on $G, I F$ is the augmentation ideal of $F$, and $\left(R^{\text {ab }}\right)^{n}$ is the $n$-fold tensor product of $R^{\text {ab }}$ over $\mathbf{Z}$ (see [8, Chapter VI, section 13]). The function $N \rightarrow \mathbf{Z} G \otimes_{F} I F, n \mapsto 1 \otimes(\tilde{n}-1)$ induces a homomorphism $\gamma_{1}: N /[G, N]$ $\rightarrow H_{1}\left(\operatorname{ker} \phi_{\#}\right)$. For each $x$ in $G \wedge N$ choose an $\tilde{x}$ in $F \wedge S$ such that the canonical map $F \wedge S \rightarrow G \wedge N$ maps $\tilde{x}$ onto $x$, and let $\partial: F \wedge S \rightarrow S$ be the map $f \wedge s \rightarrow[f, s]$. The function $\pi_{3}(G \wedge N) \rightarrow \mathbf{Z} G \otimes_{\mathbf{Z}} R^{\text {ab }}, \quad x \mapsto 1 \otimes(\partial \bar{x})[R, R]$ induces a homomorphism $\gamma_{2}: \pi_{3}(G \wedge N) \rightarrow H_{2}\left(\operatorname{ker} \phi_{\#}\right)$. We leave the reader to check that the homomorphisms $\gamma_{1}, \gamma_{2}$ are well defined, and that the resulting diagram is commutative (use [8, Chapter VI] to obtain explicit descriptions of the isomorphisms $\alpha_{i}, \beta_{i}$ ). It follows that $\gamma_{1}$ and $\gamma_{2}$ are isomorphisms by the 5-lemma because $\alpha_{i}, \beta_{i}$ are all isomorphisms.

3. Remarks. 1. Suppose the group $G$ contains two normal subgroups $M, N$ such that $G=M N$. In [3] a homotopy push-out

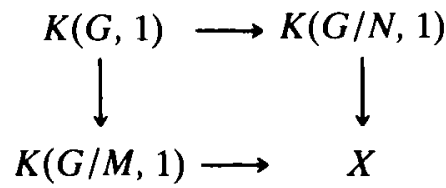

of three $K(\pi, 1)$-spaces is considered. It is shown that $H_{3}(X)=\pi_{3}(M \wedge N)$ and $H_{2}(X)=$ $M \cap N /[M, N]$. Part of the Mayer-Vietoris sequence associated to the push-out is thus the exact sequence:

$$
\begin{aligned}
& H_{3}(G) \rightarrow H_{3}(G / M) \oplus H_{3}(G / N) \rightarrow \pi_{3}(M \wedge N) \rightarrow H_{2}(G) \rightarrow \\
& H_{2}(G / M) \oplus H_{2}(G / N) \rightarrow M \cap N /[M, N] \rightarrow H_{1}(G) \rightarrow \\
& \quad H_{1}(G / M) \oplus H_{1}(G / N) \rightarrow 0 .
\end{aligned}
$$

The first six terms of (12) (starting from the right) are derived algebraically in [4]. Note that on taking $M=G$ the above sequence (1) is retrieved.

2. By omitting relation (7) in the above definition of the exterior product $M \wedge N$, we obtain the notion of a non-abelian tensor product $M \otimes N$ (this notion is related to a notion of [10] and described more generally in [2]). Since $\pi_{3}(M \wedge N)$ is a quotient of the kernel of the "commutator map" $\delta: M \times N \rightarrow M \cap N$, it is clear from Theorem 7 that a short exact sequence of groups

$$
1 \rightarrow N \rightarrow G \rightarrow Q \rightarrow 1
$$


gives rise to a six term exact sequence in homology

$$
\operatorname{ker}(G \otimes N \stackrel{\delta}{\rightarrow} N) \rightarrow H_{2}(G) \rightarrow H_{2}(Q) \rightarrow N /[G, N] \rightarrow H_{1}(G) \rightarrow H_{1}(Q) \rightarrow 0 .
$$

If $N$ is central in $G$, then $\operatorname{ker}(G \otimes N \stackrel{\delta}{\rightarrow} N)$ is isomorphic to the usual tensor product $G^{\text {ab }} \otimes N$ of abelian groups. The term $G^{\text {ab }} \otimes N$ is often referred to as the "Ganea term" $[6,10]$.

3. In [4] the construction of a non-abelian exterior product of Lie algebras is given, and used to obtain the first six terms of the Lie algebra analogue of the exact sequence (12).

Acknowledgement. The author would like to thank Prof. R. Brown for his helpful comments on earlier drafts of this work, and the S.E.R.C. for support in 1981-84.

\section{REFERENCES}

1. R. Brown, Coproducts of crossed $P$-modules: applications to second homotopy groups and to the homology of groups, Topology 23 (1984), 337-345.

2. R. Brown and J.-L. Loday, Excision homotopique en basse dimension, C.R. Acad. Sci. Paris Sér I Math. 298 (1984), 353-356.

3. R. Brown and J.-L. Loday, Van Kampen theorems for diagrams of spaces, to appear in Topology.

4. G. J. Ellis, Crossed modules and their higher dimensional analogues, (University of Wales Ph.D. Thesis, 1984).

5. B. Eckmann and P. J. Hilton, On central group extensions and homology, Comment. Math. Helv. 46 (1971), 345-355.

6. B. Eckmann, P. J. Hilton and U. Stammbach, On the homology theory of central group extensions: the commutator map and stem extensions, Comment. Math. Helv. 47 (1972), 102-122.

7. A. Gut, A ten term exact sequence in the homology of a group extension, J. Pure Appl. Algebra 8 (1976), 243-260.

8. P. J. Hilton and U. Stammbach, A course in homological algebra, Graduate Texts in Mathematics 4 (Springer-Verlag, 1970).

9. J.-L. Loday, Spaces with finitely many non-trivial homotopy groups, J. Pure Appl. Algebra 24 (1982), 179-202.

11. C. Miller, The second homology group of a group, Proc. Amer. Math. Soc. 3 (1952), $588-595$.

10. A. S.-T. Lue, The Ganea map for nilpotent groups, J. London Math. Soc. (2) 14 (1976),

Department of Pure Mathematics

University College of North Wales

BANGOR

GWYNEDD LL57 2UW

U.K. 